\title{
Vortex in Chiral Superconducting State
}

\author{
J. Goryo \\ Department of Physics, Hokkaido University, \\ Sapporo, 060-0810 Japan \\ E-mail: goryo@particle.sci.hokudai.ac.jp
}

\begin{abstract}
We have investigated the vortex in chiral superconductors, especially in $p$-wave case. In chiral superconductors the Cooper pair has orbital angular momentum hence $U(1)$, parity $(\mathrm{P})$ and time reversal symmetry $(\mathrm{T})$ are broken simultaneously. We have found that the vortex has fractional charge and fractional angular momentum which comes from P- and T-violation. The fractionalization of the angular momentum suggests that the vortex could be anyon which obeys the fractional statistics. We have also pointed out that the electric field is induced near the vortex core and non-trivial electromagnetic phenomena are expected to occur.
\end{abstract}

In unconventional superconductivity, it has been pointed out the possibility of the existence of chiral ( $\mathrm{P}$ - and T-violating) superconducting states, in which the condensation of Cooper pairs that have orbital angular momentum occurs. 6 Recently, it is argued the realization of the chiral $p$-wave $\left(p_{x}+i p_{y}\right.$-wave $)$ superconductivity in $\mathrm{Sr}_{2} \mathrm{RuO}_{4}$. 2 Chiral $d$-wave $\left(d_{x^{2}} \mathrm{q}^{2}+i d_{x y}\right.$-wave) superconductivity has been proposed in High- $\mathrm{T}_{c}$ superconductors 3 It is important to investigate $\mathrm{P}$ - and T-violating phenomena to probe the chiral superconductivity. In the present paper, we investigate the vortex in chiral superconductors, especially in $p$-wave case, and show that it has fractional charge and fractional angular momentum as the consequence of $\mathrm{P}$ - and $\mathrm{T}$-violation.4

$\mathrm{Sr}_{2} \mathrm{RuO}_{4}$ has a layered perovskite crystal structure without cupper and has low transition temperature $T_{c} \simeq 1.5[K]$, and is a strongly correlated 2-dimensional Fermi liquid in normal state. Rice and Sigrist proposed that pairing symmetry of orbital part is chiral $p$-wave, which has the same orbital symmetry of superfluid ${ }^{3} \mathrm{He}-\mathrm{A}$.6 The proposal is consistent with the discovery of internal magnetic field in the superconducting phase by $\mu \mathrm{SR}$ measurement and the experiment of ${ }^{17} \mathrm{O}-\mathrm{NMR}$ Knight shift. In the chiral $p$-wave superconductor, Cooper pair has orbital angular momentum $l_{z}=1$, where $z$ is perpendicular to the superconducting plane, i.e., the same direction as the c axis of the crystal. Therefore, local $U(1)$ gauge symmetry, $\mathrm{P}$ and $\mathrm{T}$ are spontaneously broken by the same order parameter.

The above situation is analogous to the quantum Hall system (QHS), which is the 2-dimensional electron system in an external magnetic field. In QHS the external magnetic field violates $\mathrm{P}$ and $\mathrm{T}$, but the explicit local $U(1)$ gauge symmetry is preserved. It was shown that the Chern-Simons term is jnduced in the effective action of the gauge field when Fermion is integrated out $\mathrm{O}$ The Chern-Simons term is a $\mathrm{P}$ - and T-odd bi-linear form of the gauge fields and has one derivative. Therefore, this term plays important roles in low energy and long distance physics and causes $\mathrm{P}$ - and T-violating electromagnetic phenomena. The coefficient of the induced Chern-Simons term is quantized exactly as (integer) $\times \frac{e^{2}}{2 \pi} . \quad U(1)$ gauge invariance guarantees the exact quantization. Since the coefficient of the term be- 
comes the Hall canductance, the integer quantum Hall effect was explained from gauge invariance. 89

It was shown that the Chern-Simons term is also induced in the GinzburgLandau action of chiral $R$-wave superconductors by integrating out Fermion and $U(1)$ Goldstone mode . 10 The term has a form written as

$$
\int d^{3} x \frac{\sigma_{x y}}{2} \varepsilon^{i j}\left(A_{0}^{\mathrm{T}} \partial_{i} A_{j}+A_{i} \partial_{j} A_{0}^{\mathrm{T}}\right) .
$$

$A_{0}^{\mathrm{T}}$ is the transversal component of $A_{0}$ written as $A_{0}^{\mathrm{T}}=A_{0}-\frac{\partial_{0}}{\partial_{0}^{2}-c_{s}^{2} \partial^{2}}\left(\partial_{0} A_{0}-c_{s}^{2} \partial \cdot \mathbf{A}\right)$. In this case, the Chern-Simons term does not have exactly quantized coefficient $\sigma_{x y}$ written as

$$
\sigma_{x y}=\left.\frac{i}{2 !} \varepsilon^{i j} \frac{\partial}{\partial q^{j}} \pi_{0 i}(q)\right|_{q=0}=\frac{e^{2}}{8} \int \frac{d^{2} p}{(2 \pi)^{2}} \frac{\operatorname{tr}\left[\vec{g} \cdot(\partial \vec{g} \times \partial \vec{g})-g_{3}(\partial \vec{g} \times \partial \vec{g})_{3}\right]}{\operatorname{tr}\left[\frac{1}{2} \vec{g} \cdot \vec{g}\right]^{\frac{3}{2}}},
$$

here $\pi_{0 i}(q)$ is the density-current correlation function, $t r$ means trace about real spin indices and $\partial=\frac{\partial}{\partial \mathbf{p}} . \vec{g}(\mathbf{p})$ is related to Fermion propagator $G\left(p_{0}, \mathbf{p}\right)=$ $\left(p_{0}-\vec{g}(\mathbf{p}) \cdot \vec{\tau}\right)^{-1}$ in Bogoliubov-Nambu Fermion representation $\Psi_{\alpha}=\left(\begin{array}{c}\psi \\ \psi^{\dagger}\end{array}\right)$ with isospin $\alpha=1,2$, and $\vec{\tau}$ is $2 \times 2$ Pauli matrices with isospin indices. The first term in Eq.(2) is a topological invariant. Volovik have argued this type of topological invariant. 11 The second term is not a topological invariant. The second term appears because $U(1)$ gauge symmetry is spontaneously broken 10 Typical $\vec{g}$ for chiral $p$-wave superconductor with 1 band circular Fermi surface is written as

$$
\vec{g}(\mathbf{p})=\left(\begin{array}{c}
\left|\eta_{0}\right| \hat{p_{x}} i \sigma_{3} \sigma_{2} \\
-\left|\eta_{0}\right| \hat{p_{y}} i \sigma_{3} \sigma_{2} \\
\frac{\mathbf{p}^{2}}{2 m}-\epsilon_{\mathrm{F}}
\end{array}\right),
$$

$\vec{\sigma}$ is $2 \times 2$ Pauli matrices with real spin indices. By substituting Eq. (3) into Eq. (2), we obtain the value

$$
\sigma_{x y}=\frac{e^{2}}{4 \pi}
$$

which coincides with the fine structure constant. In this case, the non-topological term in Eq. (2) vanishes accidentally. In general, the non-topological term does not vanish. Actually, if we calculate $\sigma_{x y}$ in the tight binding scheme, the nontopological term has negligibly small value and $\sigma_{x y}$ is approximately equal to the fine structure constant.10 The existence of the Chern-Simons term suggests that a Hall effect without magnetic field occurs 1011

Next we consider a vortex in chiral $p$-wave superconductor. Except for the Chern-Simons term, the quite general phenomenological Ginzburg-Landau free energy of the chiral $p$-wave superconductor has been proposed by Sigrist and Uedat with tetragonal symmetry which corresponds to the layered perovskite structure of the crystal lattice. We combine both of them and investigate the vortex solution. The vortex charge is defined by the Gauss law which is modified by the Chern-Simons term and it is calculated as

$$
Q=\int d^{2} x\left\{\nabla \cdot \mathbf{E}-\sigma_{x y} \mathrm{~B}\right\}=-\sigma_{x y} \frac{2 n \pi}{2 e}=-\frac{n e}{4},
$$


because the electric field is screened. $n=0, \pm 1, \pm 2 \cdots$ is the vorticity. We can see the flux $\frac{2 n \pi}{2 e}$ is attached to the charge, i.e. the vortex is an object such as fluxcharge composite and analogous to Laughlin's quasiparticle in fractional quantum Hall state. It was argued by many authors that the change in the density of electrons in the vortex core due to the spatial dependence of the orderparameter also gives the charge of the vortex 12 and we call it "the regular charge". Recently, Volovik pointed out that the occupation of the zero-energy bound states of electrons, which exist in the vortex core of the chiral p-wave superconductor 133 could contributes the charge of the vortex. 14.15 We call it "zero-mode charge". It is important how to distinguish these two charges and the fractional charge Eq. (5) that comes from the Chern-Simons term. It can be done, even in the case that the regular charge or the zero-mode charge are of order $e$, by comparing the vortex charges of vorticities $n=1$ and $n=-1$ because the fractional charge Eq. (5) changes its sign but other two charges do not depend on the sign of vorticity. The definition of the vortex angular momentum is

$$
J=\int d^{2} x \mathbf{r} \times \mathcal{P} .
$$

$\mathcal{P}$ is the momentum density which is defined as the generator of the translation. We find $J$ is a integral of the total derivative terms of the gauge fields and proportional to $\sigma_{x y}$. $J$ takes the fractional value such as

$$
J=-\frac{n^{2}}{16}
$$

Since the charge and the angular momentum are proportional to $\sigma_{x y}$, we see that the Chern-Simons term creates these non-zero fractional values. We have found that these values are topological and depends only on asymptotic behavior of the gauge fields, hence they do not depend on the form of Ansatz and also do not depend on the phenomenological parameters in Ginzburg-Landau theory. Therefore, the results Eq. (5) and Eq. (7) would be valid for realistic systems such as superconducting $S_{2} R u O_{4}$. A detailed discussion is given in Ref. 1 . Evidence that the vortex we consider is a candidate of anyon can be seen in the fact that vortex has fractional angular momentum.16 It is expected that transmutation of the statistics occurs. The further discussion is needed to see the fractional statistics of these vortices experimentally. By using a symple Ansatz, we found a spatial dependence of a radial electric field numerically. The electric field exists around the core in the region $r<\lambda$ ( $\lambda$ London penetration depth) and its magnitude is about 1 Volt/meter.

In summary, we have discussed a vortex in chiral $p$-wave superconductor , where $U(1), \mathrm{P}$ - and T-symmetry are broken simultaneously. The realization of such a superconductivity is argued in $\mathrm{Sr}_{2} \mathrm{RuO}_{4}$. We have investigated the vortex based on the Ginzburg-Landau Lagrangian. The Ginzburg-Landau Lagrangian of the system contains the Chern-Simons term. We have found that the vortex has fractional charge $-\frac{n e}{4}$ and fractional angular momentum $-\frac{n^{2}}{16}$ which come from the existence of the Chern-Simons term, i.e. P- and T-violation of the ground state. These values are topologically stable and do not depend on the form of Ansatz and the phenomenological parameters in the Ginzburg-Landau Lagrangian. Following the discussion in Ref.16, the vortex could obey the fractional statistics. We have also 
investigated the electromagnetic property of the vortex. We have found that the electric field is induced by the Chern-Simons term near the vortex core. It is interesting if these exotic feature is observed experimentally.

Our discussion is valid for other chiral superconductors, such as "anisotropic chiral $p$-wave" superconductor in which the symmetry of the wave function of Cooper pair is $\left(\sin p_{x}+i \sin p_{y}\right)$-wave 17 and $d_{x^{2}-y^{2}}+i d_{x y}$-wave superconductor, since it has been shown that the Chern-Simons term is also induced in the Ginzburg-Landau Lagrangian of these superconductors. 10

The author is grateful to K. Ishikawa, N. Maeda, Y. Maeno, M. Matsumoto, Y. Okuno, M. Sigrist, and G.E. Volovik for useful discussions.

\section{References}

1. M. Sigrist and K. Ueda, Rev. Mod. Phys. 63239 (1991).

2. Y. Maeno, et. al., Nature 372 532-534 (1994); T. M. Rice and M. Sigrist, J. Phys.: Condensed Matter 7 (1995) L643.

3. R. B. Laughlin, Phys. Rev. Lett. 805188 (1998).

4. J. Goryo, cond-mat/9908113.

5. J. Hong, Y.Kim and P.Pac, Phys. Rev. Lett. 64, 2230 (1990); R.Jackiw and E.Weinberg, ibid 642234 (1990); G. E. Volovik, AIP Conference Proceedings 194, Quantum Fluids and Solids - 1989, eds. G.G.Ihas and Y.Takano, Gainesville, Fl. 1989, 136-146.

6. See, for example, G. E. Volovik, "Exiotic Property of superfluid ${ }^{3} H e$ ", World Scientific, Singapole - New Jersey - London - Hong Kong, (1992).

7. G. M. Luke et. al., Nature 394, 558-561 (1998); K. Ishida, et. al., Nature 396, 658 (1998).

8. K. Ishikawa, Phys. Rev. Lett. 53, 1615 (1984). N. Imai, et. al., Phys. Rev. B42, 10610 (1990);

9. D. J. Thouless, M. Kohmoto, M.P. Naightingale, and M. den Nijs, Phys. Rev. Lett. 49, 405 (1982).

10. J. Goryo and K. Ishikawa, Phys. Lett. A 260, 294 (1999) ; J. Phys. Soc. Jpn. 67, 3006 (1998); Phys. Lett. A. 246549 (1998).

11. G.E. Volovik, Sov. Phys. JETP 67, 1804 (1988); JETP Lett. 66, 522 (1997).

12. See, for example, D. I. Khomskii et. al., Phys. Rev. Lett. 751384 (1995);

13. N. B. Kopnin and M. M. Salomaa, Phys. Rev. B 449667 (1991); M. Matsumoto and M. Sigrist, J. Phys. Soc. Jpn. 68724 (1999).

14. G. E. Volovik, cond-mat/9909426 (accepted in JETP Lett.).

15. R. Jackiw and C. Rebbi, Phys. Rev. D 133398 (1976).

16. F.Wilczek, Phys. Rev. Lett. 49957 (1982).

17. K. Miyake and O. Narikiyo, Phys. Rev. Lett. 831423 (1999). 